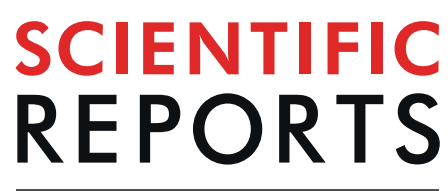

natureresearch

\title{
An Ultrasonically Powered Implantable Microprobe for Electrolytic Ablation
}

\author{
A. Kim ${ }^{1 *}$, S. K. Lee ${ }^{2}$, T. Parupudi ${ }^{3,4}$, R. Rahimi ${ }^{3,4}$, S. H. Song ${ }^{5}$, M. C. Park ${ }^{1}$, S. Islam ${ }^{1}{ }^{1}$, \\ J. Zhou ${ }^{3,4}$, A. K. Majumdar', J. S. Park ${ }^{6}$, J. M. Yoo ${ }^{7}$ \& B. Ziaie ${ }^{3,4^{*}}$
}

Electrolytic ablation (EA) is a promising nonthermal tumor ablation technique that destroys malignant cells through induction of a locoregional pH change. EA is typically performed by inserting needle electrodes inside the tumor followed by application of direct current (DC), thus inducing electrolysis and creating localized $\mathrm{pH}$ changes around the electrodes. In this paper, we report an ultrasonically powered implantable EA microprobe that may increase the clinical relevance of EA by allowing wireless control over device operation (capability to remotely turn the device on and off) and providing flexibility in treatment options (easier to administer fractionated doses over a longer period). The wireless EA microprobe consists of a millimeter-sized piezoelectric ultrasonic receiver, a rectifier circuit, and a pair of platinum electrodes (overall size is $9 \times 3 \times 2 \mathrm{~mm}^{3}$ ). Once implanted through a minimally invasive procedure, the microprobe can stay within a solid tumor and be repeatedly used as needed. Ultrasonic power allows for efficient power delivery to $\mathrm{mm}$-scale devices implanted deep within soft tissues of the body. The microprobe is capable of producing a direct current of $90 \mu \mathrm{A}$ at a voltage of $5 \mathrm{~V}$ across the electrodes under low-intensity ultrasound $\left(-200 \mathrm{~mW} / \mathrm{cm}^{2}\right)$. The $\mathrm{DC}$ power creates acidic $(\mathrm{pH}<2)$ and alkaline $(\mathrm{pH}>12.9)$ regions around the anode and the cathode, respectively. The $\mathrm{pH}$ change, measured using tissue-mimicking agarose gel, extends to $0.8 \mathrm{~cm}^{3}$ in volume within an hour at an expansion rate of $0.5 \mathrm{~mm}^{3} / \mathrm{min}$. The microprobe-mediated EA ablative capability is demonstrated in vitro in cancer cells and ex vivo in mouse liver.

Surgical resection remains the first therapeutic option for early-stage solid tumors. In advanced metastatic situations, systemic chemotherapy or radiation therapy is typically used to control disease progression. Local thermal ablation using insertable RF or microwave probes has also been used to destroy solid tumors in cases where the tumor is deemed to be nonresectable (e.g., liver or pancreatic tumors close to a sensitive anatomical structure $)^{1}$. Although clinically accepted, thermal ablation methods face limitations in cases where the tumor is located near a major blood vein, for which collateral thermal damage is unacceptable ${ }^{1-3}$. Other ablation methods using laser sources, high-intensity focused ultrasound (HIFU), and cold (cryo-ablation) have also been proposed and are currently at various stages of development and clinical trials ${ }^{4-6}$. Electrolytic ablation (EA), a nonthermal method in which a localized $\mathrm{pH}$ region is created via two electrodes inserted into a tumor and connected to a direct current (DC) source, is an attractive method that can controllably treat the tumor and limit the damage to adjacent critical structures (due to the use of nonextreme energy) ${ }^{2,7-9}$. EA was first used to treat lung tumors ${ }^{10}$ followed more recently by its application to liver $^{7}$ and pancreatic tumors 8 . Both thermal and electrolytic methods require the insertion of probes into the tumor under image-guided procedures. Although repeated therapy sessions are preferred and common due to the strongly resistant nature of tumors, each session of cancer treatment, such as chemo- or radiotherapy is usually administered through difficult and cumbersome in-patient procedures ${ }^{11-14}$.

In this paper, we present an ultrasonically powered implantable microprobe that enables wireless EA therapy. Figure 1a illustrates a schematic of the microprobe implanted in a tumor using a procedure similar to the

${ }^{1}$ Department of Electrical and Computer Engineering, Temple University, Philadelphia, PA, 19122, USA. ${ }^{2}$ Jubilee Biotechnology LLC, Philadelphia, PA, 19122, USA. ${ }^{3}$ School of Electrical and Computer Engineering, Purdue University, West Lafayette, IN, 47907, USA. 'Birck Nanotechnology Center, West Lafayette, IN, 47907, USA. ${ }^{5}$ Department of Electronic Engineering, Sookmyung Women's University, Seoul, South Korea. ${ }^{6}$ Pancreatobiliary Cancer Clinic, Department of Surgery, College of Medicine, Gangnam Severance Hospital, Yonsei University, Seoul, South Korea. ${ }^{7}$ Department of Microbiology, School of Medicine, CHA University, Seongnam, South Korea. *email: albertkim@temple.edu; bziaie@purdue.edu) 
a

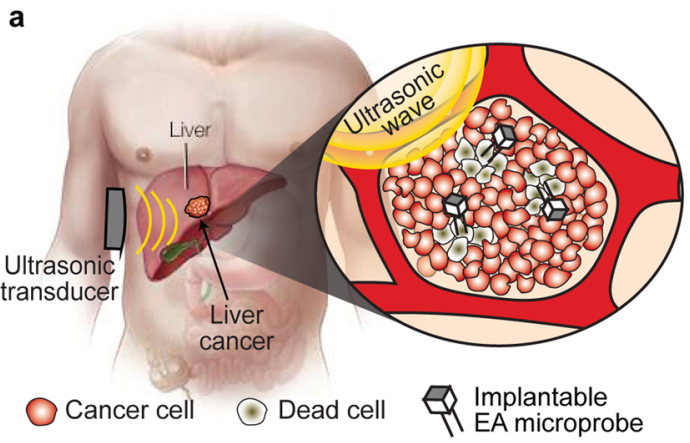

C

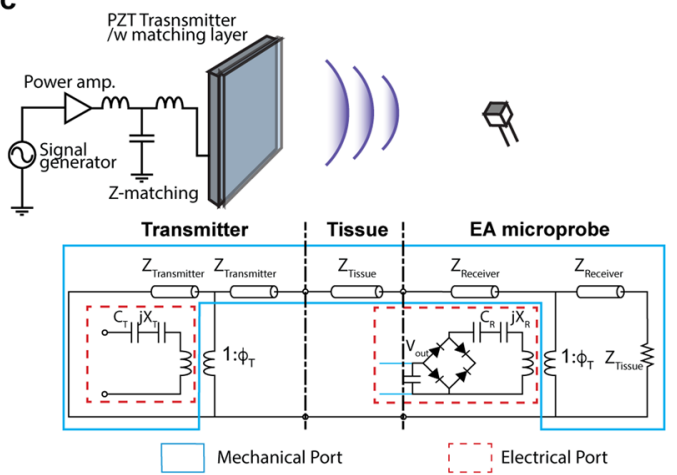

b

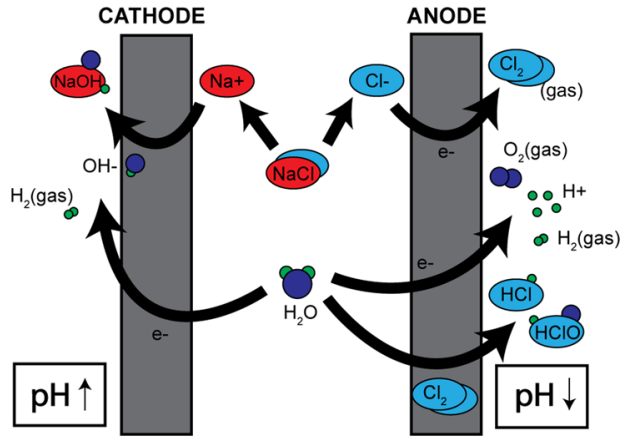

d
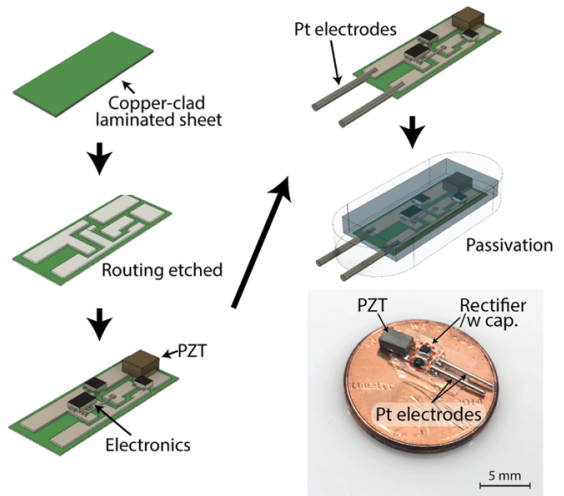

Figure 1. (a) Schematic view of wireless electrolytic ablation therapy. Multiple EA microprobes can be implanted inside a tumor and powered remotely by an ultrasonic wave. (b) Essential chemical reactions occurring during EA: anode: $\mathrm{Cl}_{2}$ (gas), $\mathrm{H}_{2}$ (gas), $\mathrm{O}_{2}$ (gas), $\mathrm{HCl}, \mathrm{HClO}$; cathode: $\mathrm{NaOH}, \mathrm{OH}^{-}, \mathrm{H}_{2}$ (gas).

(c) Ultrasonic power: schematics of the power transfer link and its theoretical electromechanical model ${ }^{38}$. (d) Fabrication process: 1. circuit is drawn on copper-clad laminated polyimide sheet (Pyralux), 2. circuit layout lithography and etching, 3. soldering components, 4. platinum electrode assembly, 5. passivation and optional packaging, and 6. a photograph of the $\mathrm{mm}$-scale electrolytic-ablation device $(9 \mathrm{~mm} \times 5 \mathrm{~mm} \times 2 \mathrm{~mm})$.

deployment of brachytherapy seeds. The device is powered from outside of the body using ultrasound and subsequently delivers direct current to the tumor, inducing an in situ local $\mathrm{pH}$ change. Ultrasonic power allows the device to be miniaturized to dimensions ( $\mathrm{mm}$-scale) that are not feasible using traditional inductive wireless powering methods ${ }^{15}$. In addition, ultrasound has a long operation range $(>10 \mathrm{~cm})^{15-20}$ and a higher power transfer efficiency than traditional inductive power at $\mathrm{mm}$-scale reviver sizes ${ }^{15}$, and it is significantly less sensitive to angular misalignment between the transmitter and the receiver ${ }^{15,17,20-23}$. The implantable microprobe may increase the clinical relevance of EA by allowing wireless control over device operation (capability to remotely turn the device on and off) and providing flexibility in treatment options (easier to administer fractionated doses over a longer period). Although the described microprobe requires initial insertion using a biopsy needle or a trocar, once in place, it can be remotely reactivated if further treatments are necessary without repeated insertion of needle electrodes.

\section{Results and Discussion}

Electrolytic ablation principles. Figure $1 \mathrm{~b}$ illustrates the essential electrochemical reactions during in situ local pH modulation with low-voltage DC-induced electrolysis. The primary effect of electrolysis is the generation of hydrogen (hydronium) and hydroxide ions (anode: $2 \mathrm{H}_{2} \mathrm{O} \leftrightarrow \mathrm{O}_{2}+4 \mathrm{H}^{+}+4 e^{-}$and $2 \mathrm{Cl}^{-} \rightarrow \mathrm{Cl}_{2}+2 e^{-}$; cathode: $\left.2 \mathrm{H}_{2} \mathrm{O}+2 e^{-} \leftrightarrow \mathrm{H}_{2}+2 \mathrm{OH}^{-}\right)^{8}$. A secondary effect of electrolysis involves sodium chloride $(\mathrm{NaCl})$, which forms $\mathrm{Na}^{+}$ions that move toward the cathode and $\mathrm{Cl}^{-}$ions that move toward the anode. These ions react with the aqueous in vivo environment to produce sodium hydroxide and hydrogen at the cathode $\left(2 \mathrm{NaCl}+2 \mathrm{H}_{2} \mathrm{O} \leftrightarrow\right.$ $\left.\mathrm{Cl}_{2}+\mathrm{H}_{2}+2 \mathrm{NaOH}\right)$ and hydrochloric acid, oxygen, and chlorine at the anode $\left(2 \mathrm{Cl}^{-} \leftrightarrow \mathrm{Cl}_{2}+2 e^{-}\right.$and $\mathrm{Cl}_{2}+\mathrm{H}_{2} \mathrm{O} \leftrightarrow$ $\left.\mathrm{HClO}+\mathrm{H}^{+}+\mathrm{Cl}^{-}\right)$. As a result of reactions, the region surrounding the anode becomes acidic $(\mathrm{pH}<6)$, whereas the region around the cathode becomes alkaline $(\mathrm{pH}>9)$. This localized $\mathrm{pH}$ change can cause cell death around the electrodes.

Wireless ultrasonic powering. An ultrasonic power link consists of three elements: an ultrasonic transmitter, soft tissue, and a receiver (microprobe in our case) (Fig. 1c-top). The microprobe converts incoming ultrasonic waves into a direct electrical current and induces electrolysis through a pair of platinum electrodes in the tumor. (Fig. 1c-bottom) illustrates the theoretical ultrasonic power schematic equivalent circuit using the Krimholtz, Leedom, Mattaei (KLM) model (detailed theoretical discussion of the KLM model is provided in the Supplementary Information) ${ }^{24}$. 


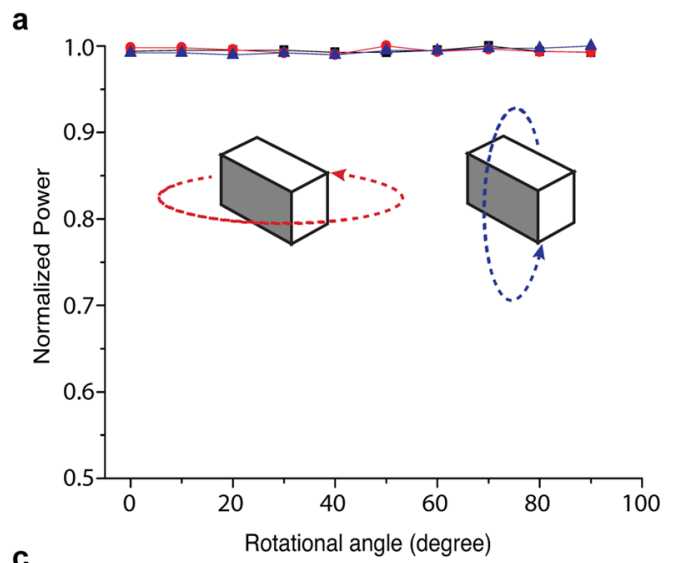

b

C

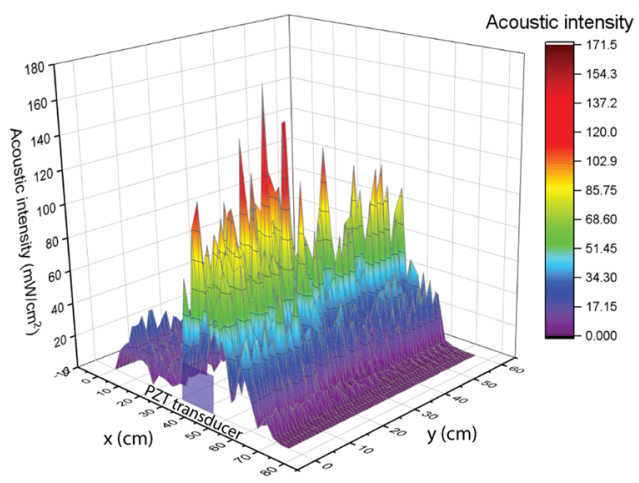

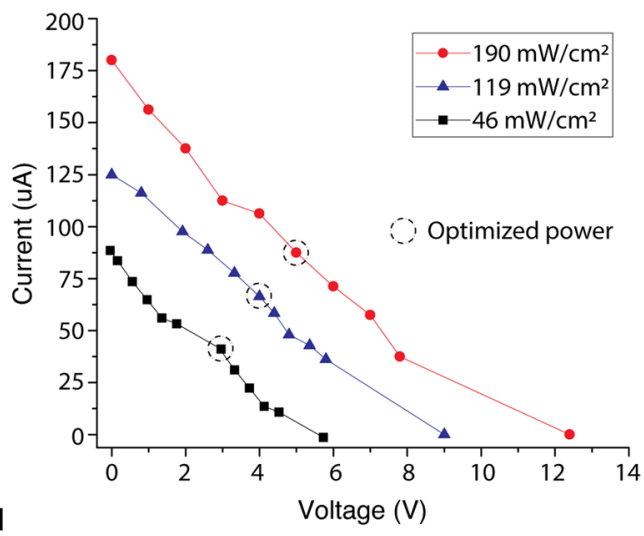

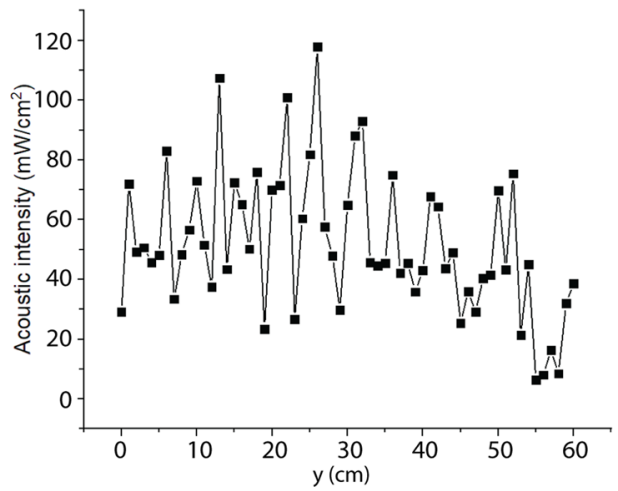

Figure 2. Wireless ultrasonic power: (a). Transverse and axial rotational angular misalignment, (b). I-V characteristics at $190 \mathrm{~mW} / \mathrm{cm}^{2}$ ultrasonic intensity, (c). Nonreflected ultrasound propagation across the frontal region of the ultrasonic transducer, (d) Distribution of ultrasonic intensity seen on the y-axis.

Prior to testing the efficacy of the microprobe, we investigated ultrasonic power for the intended microprobe form factor (the receiver size of $2 \times 4 \times 2 \mathrm{~mm}^{3}$; see Methods section for detail) (Fig. 1d). Ultrasonic power for the microprobe was characterized in terms of angular alignment sensitivity between a transmitter and a receiver, current-voltage (I-V) characteristics, and the field of operation. Figure 2 a shows the normalized received electrical power as a function of transverse and axial rotational angular misalignment measured under open-circuit conditions. The received output remained constant over $90^{\circ}$ rotations, confirming the misalignment insensitivity $^{15}$. The I-V characteristics of the microprobe were also measured to estimate the output power at three different ultrasonic intensities: 46,119 , and $190 \mathrm{~mW} / \mathrm{cm}^{2}$, corresponding to mild, moderate and strong ultrasonic intensities, respectively (Fig. 2b). The electrical power from the microprobe with platinum electrodes was measured to be $164 \mu \mathrm{W}(\mathrm{V}=3.5 \mathrm{~V}, \mathrm{I}=47 \mu \mathrm{A}), 300 \mu \mathrm{W}(\mathrm{V}=4.23 \mathrm{~V}, \mathrm{I}=71 \mu \mathrm{A})$, and $450 \mu \mathrm{W}(\mathrm{V}=5 \mathrm{~V}, \mathrm{I}=90 \mu \mathrm{A})$ under mild, moderate, and strong ultrasonic intensities, respectively. These results confirmed that sufficient power can be supplied to initiate electrolysis (the wireless power transfer efficiencies were $\left.2.7 \%, \eta=\left|P_{\text {output }} / P_{\text {input }}\right|\right)^{23}$.

The field of operation, a critical factor that determines the ablation zone and the efficacy of the treatment, was also evaluated. We measured the acoustic intensities in an arbitrary volume $\left(60 \mathrm{~cm}^{3}\right)$ in front of the transmitter with a $1-\mathrm{mm}$ spatial resolution. We used a fiber optic hydrophone (FOH, Precision Acoustics, UK) ${ }^{25}$ mounted on a manipulator (XY plotter, MakeBlock Ltd.) while manually changing the Z location to characterize the field of operation under nonreflective boundary conditions by covering the water tank walls with acoustic absorption layers (Aptflex F28, Precision Acoustics, UK). Figure 2c shows a three-dimensional map of acoustic intensity, and Fig. $2 \mathrm{~d}$ shows the distribution of acoustic intensity along the traveling direction ( $y$-axis). Overall, ultrasound intensities of over $60 \mathrm{~mW} / \mathrm{cm}^{2}$ (average) were measured for distances of up to $60 \mathrm{~cm}$ away from the transducer. Additionally, the regional peak intensities tend to concentrate along the center axis of the transducer (with a measured intensity of $120 \mathrm{~mW} / \mathrm{cm}^{2}$ at a distance of $25 \mathrm{~cm}$ from the transducer). Such a long working distance is sufficient for most deeply seated solid tumors ${ }^{20,26}$.

Ablation zone estimation. Using a tissue-mimicking agarose gel with a $\mathrm{pH}$ indicator (phenol red) ${ }^{27}$, we showed that the ablation zone (treatment locality) can be easily controlled by tuning the treatment time and the input ultrasonic power. The ablation zone (evaluated by measuring the $\mathrm{pH}$ front) as a function of time is shown in Figure $3 \mathrm{c}, \mathrm{d}$. The ablation zone was estimated by measuring the area of color change by phenol red dye, red near the cathode and yellow near the anode. We observed isotropic expansion of the ablation zone at a constant rate for a given ultrasonic intensity over time. The ablation zones after $30 \mathrm{~min}$ of operation for the anode/cathode were $0.25,0.35$, and $0.6 \mathrm{~cm}^{3}$ for ultrasonic intensities of 46,119 , and $190 \mathrm{~mW} / \mathrm{cm}^{2}$, respectively. The ablation 
a

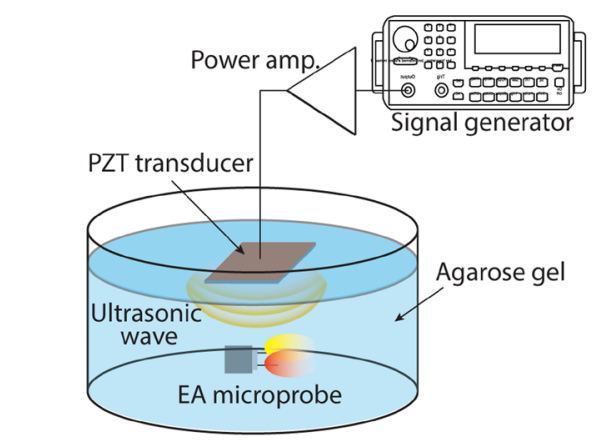

c

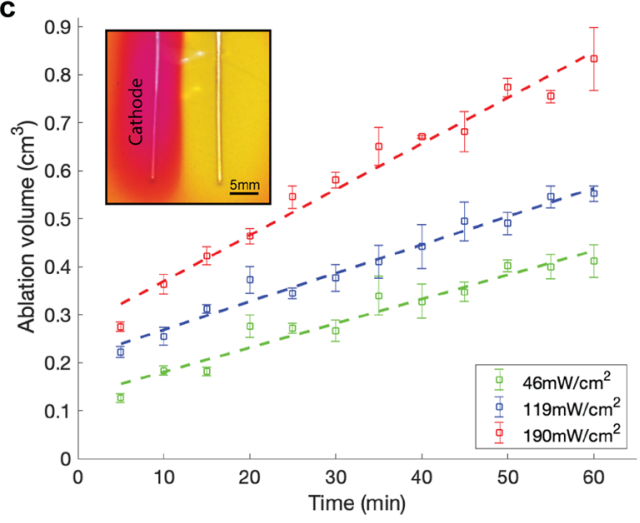

b

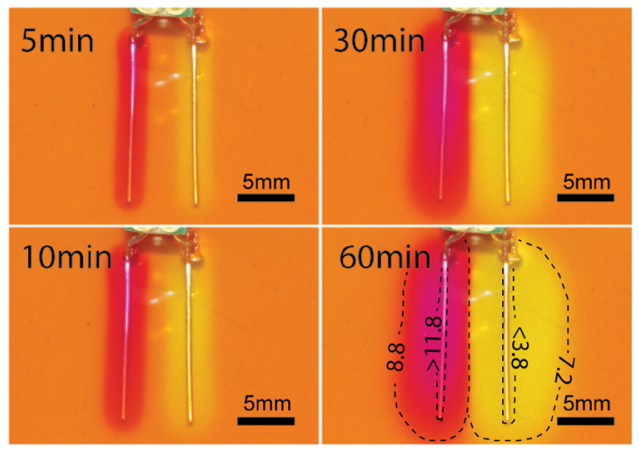

d

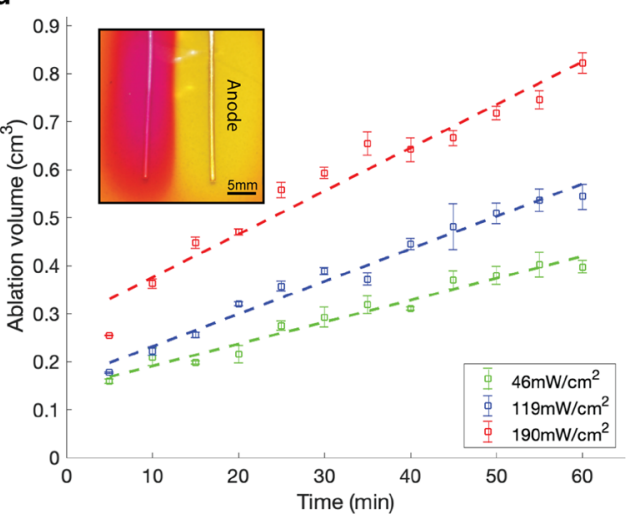

Figure 3. (a) Experimental setup used to record the in vitro ablation zone. An EA microprobe was placed in agarose gel loaded with a $\mathrm{pH}$-sensitive dye and powered by an ultrasonic transducer (figure is not to scale). (b) Time-lapse photographs showing ablation zone expansion around the cathode (left) and the anode (right). Measured $\mathrm{pH}$ values marked at concentric points around the electrodes. All scale bars: $5 \mathrm{~mm}$. (c) Progression of the area of the ablation zone ( $\mathrm{pH}$ fronts) as a function of time around the cathode and (d). Anode at three different ultrasonic intensities: 46, 119, and $190 \mathrm{~mW} / \mathrm{cm}^{2}$. Error bars represent SD.

zone volume expanded linearly from the anode/cathode as a function of time. The average rates of ablation zone expansion were respectively $0.4 / 0.5,0.7 / 0.6$, and $0.9 / 0.9 \mathrm{~mm}^{3} / \mathrm{min}$ under mild, moderate, and strong ultrasonic intensities. The $\mathrm{pH}$ front was simultaneously measured using an electrode-type $\mathrm{pH}$ meter (IC-401, Specialty Sensors LLC) in a $20 \mathrm{G}$ needle. The measured $\mathrm{pH}$ was as low as 1.7 to 3.8 near the anode and as high as 11.8 to 12.3 near the cathode (average $\mathrm{pH}$ diffusion rate was $0.09 / \mathrm{min}$ ) (Fig. $3 \mathrm{~b}$ ).

The ablation zone expansion size and rate are a function of ionic mobility and the applied electric field (similar to the electrophoresis). Although the $\mathrm{pH}$-indicated ablation zone is only an estimation and may not perfectly correspond with the distribution of cell death around the margin, it is generally agreed that EA can be designed to tailor an ablation zone based on $\mathrm{pH}$ fronts and tissue electric resistance (also related to ionic mobility). The appropriate treatment scenario must still be elucidated in animal models (e.g., using mild ultrasound with short duration to treat a region requiring extreme locality or a long single session with strong ultrasound to cover a larger volume).

In vitro validation. We evaluated the efficacy of the microprobe-mediated EA with an in vitro cell viability assay by inducing electrolysis in HMT3522 S1 breast cancer cell cultures (Fig. 4a). We used four experimental groups: (1) the microprobe group, (2) the positive control (i.e., electrolysis using a laboratory DC power source), (3) the negative control (i.e., no action), and (4) the ultrasound-only group. Each group had three cell wells, and all the experiments were repeated at least quadruple $(n \geq 4)$. The microprobe group showed significant cell death, comparable to the positive group ( $\mathrm{p}$-value $=0.5254$ ) after $30 \mathrm{~min}$ of operation (Fig. $4 \mathrm{~b}$ ). Cell death caused by the microprobe was statistically significant compared to the negative control group and ultrasound-only group (the p-value was less than 0.0001 for both). The average number of dead cells was 1,394 spots ( \pm 241 spots) in the microprobe group, 2,272 spots ( \pm 166 spots) in the positive control group, 327 spots ( \pm 78 spots) in the ultrasound-only group, and 201 spots ( \pm 5 spots) in the negative control group. Figure $4 \mathrm{~b}$ circular subsets are pictures of wells of the cell plate after Trypan blue staining.

The difference between the microprobe group and the positive control was attributed to the difference in the electrical current; the capacitive source impedance of the microprobe resulted in a lower current than the DC power supply $(105 \mu \mathrm{A}$ at $6.2 \mathrm{~V}$ from the microprobe as opposed to $1.02 \mathrm{~mA}$ at $6.2 \mathrm{~V}$ from the DC power supply, Section B). As such, the strong ultrasonic intensity $\left(190 \mathrm{~mW} / \mathrm{cm}^{2}\right)$ induced a direct current of $105 \mu \mathrm{A}$, which was slightly higher than that measured in the ablation zone experiment $(90 \mu \mathrm{A}$, Section C) due to the media difference (cell media vs. agarose). Last, the temperature was simultaneously measured throughout the experiments (infrared thermometer, Traceable ${ }^{\circledR}$ Products). We did not observe a significant temperature change $(\Delta \mathrm{T}$ was less than $1^{\circ} \mathrm{C}$ ), affirming the EA-induced cell death through the tumor microenvironment alteration ${ }^{2,9,28,29}$. 
a

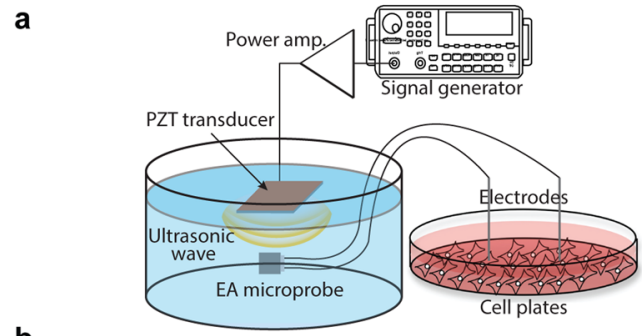

b

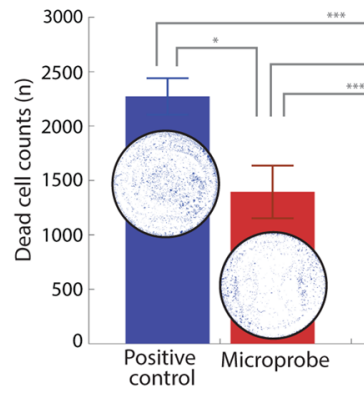

d
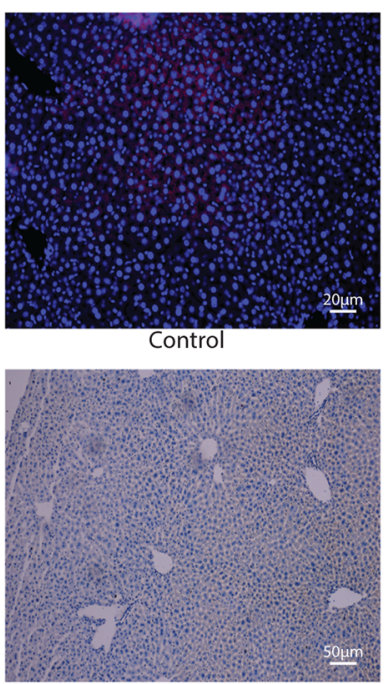

Control oinin
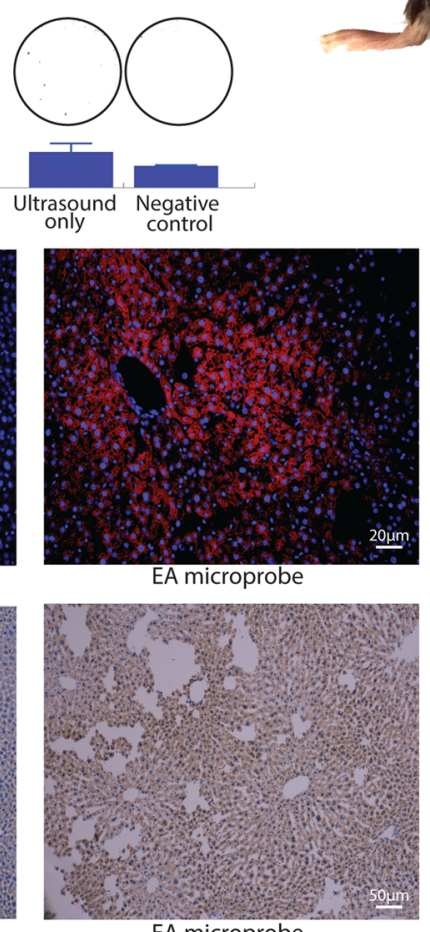

c
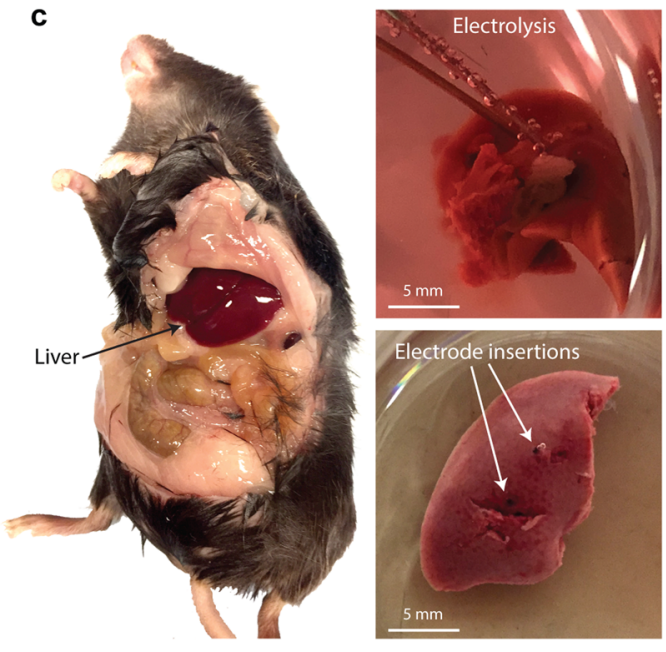

e

Figure 4. The biological action of EA microprobe: (a). Experimental setup for in vitro cell viability (figure is not to scale), (b). Cell viability assay: The microprobe is effective as a DC power source (positive control) compared to negative controls (ultrasound only and no action). (c) Ex vivo experiment using mouse liver. (d) The expression of caspase3 was detected by immunofluorescence and immunohistochemistry. Positive caspase 3 is counterstained with DAP in immunofluorescence and hematoxylin in immunohistochemistry. The control group (electrode insertion only) was compared with the experimental group. (e) Analysis of immunofluorescence: integrated density and corrected cell fluorescence were measured. Error bars represent $\mathrm{SD} ; \mathrm{n} \geq 4$; $\mathrm{p}$-value was determined using a two-tailed t-test. ${ }^{*} \mathrm{p}<0.05,{ }^{*} \mathrm{p}<0.01, * * * \mathrm{p}<0.001$.

Ex vivo validation. The ex vivo efficacy of microprobe-mediated EA was tested on fresh livers harvested from 6-month-old mice. Unambiguously demonstrated cell death is summarized in Fig. 4c. A total of two groups were prepared: a control group and a microprobe group. The control group received electrode insertion without the operation, whereas the microprobe group received strong ultrasonic intensity $\left(190 \mathrm{~mW} / \mathrm{cm}^{2}\right)$ for $30 \mathrm{~min}$. Each group had three liver samples, and all the experiments were repeated at least quadruple $(n \geq 4)$. Compared to the control group, the microprobe group showed a significant increase in cell death. Figure $4 \mathrm{~d}$ shows images of caspase 3 expression in the liver using immunofluorescence and immunohistochemistry. Positive caspase 3 was counterstained with DAPI in immunofluorescence and hematoxylin in immunohistochemistry. Total cell death was analyzed using integrated density (the product of area and mean gray value) and corrected total cell fluorescence (CTCF) (by compensating for the background) (Fig. 4e), which were statistically significant compared to the control ( $\mathrm{p}$-value $=0.0001)$; CTCFs were $409 \times 10^{3}\left( \pm 139 \times 10^{3}\right)$ and $21 \times 10^{3}\left( \pm 31 \times 10^{3}\right)$ for microprobe and control groups, respectively. Note that the output voltage from the microprobe was $6.20 \mathrm{~V}(105 \mu \mathrm{A})$, which was identical to those in the in vitro cell viability experiment.

While the ex vivo results confirmed the effectiveness of the microprobe-mediated EA, this study has limitations. The extrapolation of this study to a large animal or human may be difficult because this study mainly utilized in vitro cancer cells and mouse liver. The ablation parameters (i.e., ablation zone, treatment time, ultrasonic 


\begin{tabular}{|l|l|}
\hline Property & Value \\
\hline Longitudinal acoustic wave velocity in PZT-5A & $4,350 \mathrm{~m} / \mathrm{s}$ \\
\hline Longitudinal acoustic wave velocity in tissue & $1,540 \mathrm{~m} / \mathrm{s}$ \\
\hline Dimension of the transmitter/receiver PZT-5A & $2 \times 4 \times 2 \mathrm{~mm}^{3}$ \\
\hline Resonant frequency (measured) & $650 \mathrm{kHz}$ \\
\hline PZT dielectric constant & 1,800 \\
\hline Electromechanical coefficient $\left(k^{2}\right)$ & 0.72 \\
\hline Tissue attenuation coefficient & $\sim 2.5 \mathrm{~Np} / \mathrm{cm}^{39}$ \\
\hline
\end{tabular}

Table 1. Characteristics of the components used in the microprobe.

intensity, delivered DC output, and possible chlorine gas distribution) described herein may not be the same in a large animal or human because the larger volume of body fluid may mitigate the diffusion of $\mathrm{pH}$ and reduce (or promote) the ablation zone. Notwithstanding, we expect that the wireless controllability offered by the microprobe can be exploited to optimize the treatment for larger subjects. For example, we expect that implanting multiple microprobes can reduce the treatment time; four to five microprobes can effectively ablate a 4 -cm diameter liver tumor with an hour-long single session.

Last, we should emphasize that the microprobe requires further development and validation. Despite its small dimensions, implanted microprobes in the body should be carefully examined to evaluate their biocompatibility, long-term reliability, and efficacy. Additionally, the system may require real-time monitoring, which can be accomplished by a closed-loop system that incorporates telemetry function within the same implants ${ }^{30}$. Alternatively, one can utilize MRI to monitor the $\mathrm{pH}$ change in real-time, as demonstrated by Meir et al. ${ }^{31}$.

\section{Methods}

Fabrication of the microprobe. Figure 1d shows the fabrication procedure and the microprobe prototype. Table 1 summarizes the mechanical and electrical properties of the subcomponents of the microprobe. The microprobe is comprised of a mm-scale piezoelectric receiver element (PZT-5A, PSI-5A4E, Piezo Systems Inc., USA), a full bridge rectifier created by four surface-mount Schottky diodes (CMRSH-4DO, Central Semiconductor Corp. USA), a smoothing capacitor $(0.1 \mu \mathrm{F}, 1005 \times 8 \mathrm{R} 1 \mathrm{E} 103 \mathrm{~K} 050 \mathrm{BA}$, TDK), and a pair of platinum electrodes (platinum-clad niobium electrodes, $0.5 \mathrm{~mm}$ diameter $\times 10 \mathrm{~mm}$ length, Anomet Products, USA). The entire electronic is housed on a copper-clad polyimide sheet (Pyralux AP9141R, $100 \mu \mathrm{m}$ polymer thickness, $35 \mu \mathrm{m}$ copper thickness, DuPont, USA). The circuitry layout was fabricated using lithography followed by etching (Fig. 1d, steps 1-2). A 2 mm-thick PZT-5A plate was pre-diced to $2 \times 4 \times 2 \mathrm{~mm}^{3}$ elements using a dicing saw system (DAD-2H/6 Dicing Saw, Disco, Japan). Next, a PZT slab, a full-wave bridge rectifier, and a smoothing capacitor were substantially assembled (Fig. 1d step 3). Note that a rectifier circuit converts the PZT receiver's alternating current (AC) into direct current (DC). Then, platinum electrodes were assembled (Fig. 1d, step 4). Finally, the fully assembled microprobe was coated with $5 \mu \mathrm{m}$ Parylene- $C$ for passivation and biocompatibility (Fig. 1d, step 5). An optional custom packaging can also be added in this step. Figure $1 \mathrm{~d}$ (bottom right) shows a picture of the microprobe prototype. The overall device dimension was $9 \times 5 \times 2 \mathrm{~mm}^{3}$ with $5 \mathrm{~mm}$ active platinum electrode tips for ablation (weight $200 \mathrm{mg}$ ).

Ultrasonic power. For maximum wireless power transfer efficiency, operation must occur at the resonant frequency $(f r)$. This frequency is usually determined by physical properties, size, and aspect ratio of the piezoelectric transducers. In the 1D model, a piezoelectric transducer operates in the thickness-expansion mode, whose resonant frequency is defined by its thickness $(t)$ and wavelength $\left(f_{r}=v_{D} / 2 \times t\right.$, where $v_{D}$ is the speed of sound in the piezoelectric material). However, this is true only if the aspect ratio (width/thickness) is greater than 10 . In a cuboidal form factor, such as a receiver used in the microprobe $\left(2 \times 4 \times 2 \mathrm{~mm}^{3}\right)$, the resonant frequency shifts due to Poisson's ratio and the associated mode coupling ${ }^{19,32,33}$. Therefore, the resonant frequency of a PZT slab was measured by an impedance analyzer (Keysight E4990A, CA, USA) over a range of frequencies. The resonant frequency $(f r)$ was the point where the internal impedance was the lowest and anti-resonance $(f a)$ was the frequency where the internal impedance was the highest. The measured impedance is plotted in Supplementary Fig. 1. Several radial resonant modes were observed at lower frequencies, and the first thickness-mode resonance appeared at a frequency of $650 \mathrm{kHz}$.

All experiments were conducted in a water tank because water is a common model for soft tissue, as their acoustic impedance are similar $\left(Z_{\text {water }}=1.48 \mathrm{MRayl}, Z_{\text {tissue }} \text { avg }=1.63 \mathrm{MRayl}\right)^{34}$. A microprobe was submerged in a water tank (dimensions of $60 \times 30 \times 40 \mathrm{~cm}^{3}$ ) and positioned within the near-field range. Figure 1c (top) shows a block diagram of the ultrasonic power, which has three parts: an ultrasonic transmitter, soft tissue, and a microprobe. The left diagram indicates an external ultrasonic power system that consists of a signal generator (MSOX3024T, Keysight), a class A wideband RF power amplifier (1040 L, E\&I Ltd., NY, USA) and an impedance matching circuit connected to an ultrasonic transducer (PZT-5A, Piezo Inc., $3.6 \times 3.6 \times 2 \mathrm{~cm}^{3}$ ). The transmitting face of the PZT transducer was laid with an acoustic matching layer. The ultrasonic power utilized a continuous wave (i.e., sinusoidal wave at the resonant frequency), which allows reflection in the body and significantly reduces the angular alignment sensitivity between the transmitter and the microprobe ${ }^{23}$.

The input ultrasonic intensity was also kept below the approved FDA limit (peak intensity of $720 \mathrm{~mW} / \mathrm{cm}^{2}$ ) for the imaging application to avoid cavitation and heat-induced damage $\mathrm{e}^{35}$. All acoustic intensities were calculated from the measured peak acoustic pressure by a fiber optic hydrophone (FOH, Precision Acoustics, UK). The 
measured voltage output, $V_{F O H}(\mathrm{mV})$, from the hydrophone (monitored using an oscilloscope) was converted to peak acoustic pressure, $P_{\text {peak }}(\mathrm{MPa})$, by the following equation.

$$
P_{\text {peak }}=\frac{V_{F O H}}{\text { Sensitivity }}
$$

The calibrated sensitivity of the fiber optic hydrophone was $215.94 \mathrm{mV} / \mathrm{MPa}$. The peak acoustic pressure was then converted to the acoustic intensity, $I\left(\mathrm{~mW} / \mathrm{cm}^{2}\right)$, by the following equation:

$$
I=\frac{P_{\text {peak }}^{2}}{2 Z_{\text {water }}}
$$

The $Z_{\text {water }}$ is a specific acoustic impedance ( $\left.=1.43 \mathrm{MRayl}\right)$, which is defined as $Z=\rho \nu_{\text {Water }}$, where $\rho$ is the density $\left(\mathrm{kg} / \mathrm{m}^{2}\right)$ and $\nu_{\text {Water }}$ is the speed of sound in water $(\mathrm{m} / \mathrm{s})$. Note that the prefactor $1 / 10$ can be added to display the acoustic intensity in $\mathrm{mW} / \mathrm{cm}^{2}\left(1 \mathrm{~W} / \mathrm{m}^{2}=10 \mathrm{~mW} / \mathrm{cm}^{2}\right)$.

Ablation zone estimation. Figure 3 a shows an experimental setup for the ablation zone estimation. We used a $0.7 \%(\mathrm{w} / \mathrm{v})$ agarose gel with a $\mathrm{pH}$ indicator (i.e., phenol red) for visualization of the ablation zone. Agarose gel was used since it is electrically neutral and mechanically stable at room temperature; this gel is a well-established phantom to assess $\mathrm{EA}^{2,36,37}$. The $\mathrm{pH}$ in the agarose gel was adjusted to a physiological $\mathrm{pH}$ of 7.4 by adding $0.9 \mathrm{wt} / \%$ sodium chloride. Then, we added $1 \mathrm{ml}$ of phenol red per $100 \mathrm{ml}$ of agarose solution for colorimetric monitoring of $\mathrm{pH}$ changes. Phenol red dye turns yellow in an acidic environment and red in an alkaline environment. A central crater was carved out in the agarose gel and filled with deionized water. The microprobe was then placed in the crater, and the electrodes were inserted into the agarose gel. Time-lapse images were captured for visual tracking of $\mathrm{pH}$ fronts during the experiment (images are captured at intervals of 5 min over an hour). The images were then processed and analyzed using ImageJ software $(\mathrm{NIH})$ to measure the area of color-changed regions of the agarose gel.

Cells and cell viability staining. Figure 4a shows an experimental setup for the in vitro cell viability test. We separated the microprobe operation and electrolysis for experimental convenience. This approach also ruled out an ultrasound effect in the EA. HMT3522 S1 cells were obtained from Sigma. HMT3522 cells were cultured in DMEM supplemented with glutamine, penicillin, streptomycin, and 10\% FBS (Sigma). We first eliminated the possibility of temperature and undesired $\mathrm{pH}$ changes using $25 \mathrm{mM}$ HEPES buffer in PBS. After the microprobe operation, we used the Trypan Blue cell viability assay. After collecting supernatant cells, adherent cells were released by 6 min $0.25 \%$ Trypsin-EDTA. All dead cells were then stained with $0.4 \%$ Trypan Blue. After centrifuging and washing the pellets with PBS twice, the pellets were resuspended in a new plate. We then counted the number of stained cells, corresponding to cell death.

Ex vivo experiments and immunofluorescence/immunohistochemistry. All experiments were approved and conducted according to the regulations and guidelines of the Gangnam Severance Hospital Institutional Animal Care and Use Committee and Institutional Ethics Committee. Livers were harvested and kept in $4^{\circ} \mathrm{C}$ HBSS during the experiment. After the microprobes were inserted into the liver (Fig. 4b), ultrasonication was performed for $30 \mathrm{~min}$. Note that the distance between electrodes was $1 \mathrm{~cm}$. After the microprobe operation, livers were washed with PBS and fixed in $4 \%$ PFA O/N. The paraffin sections were deparaffinized in xylene and rehydrated using graded ethanol. Antigen retrieval was performed using unmasking solution (Vector Laboratories) in a humidified chamber. Samples were blocked with 5\% BSA and 5\% sheep serum in PBST for 1 hour at room temperature. The anti-caspase 3 antibody was incubated overnight at $4{ }^{\circ} \mathrm{C}$ (AB3623, Millipore, USA). After washing three times with PBST, Alexa 594 secondary antibodies were applied for 30 min at room temperature. The samples were then washed again and mounted with Prolong Gold antifade reagent with DAPI (Jackson). Images were taken using an Olympus BX43. For immunohistochemistry, HRP-conjugated secondary antibody was developed using DAB.

Statistical analysis. The results from independent experiments were expressed as mean \pm SD (standard deviation). Statistical analysis of all the experimental data was performed using the Student's $t$-test with JMP ${ }^{\circledR}$ Pro 14.0. All the experiments were repeated at least quadruple. Data were considered statistically significant when $P$-value is less than 0.05 .

Received: 6 March 2019; Accepted: 10 January 2020;

Published online: 30 January 2020

\section{References}

1. Knavel, E. M. \& Brace, C. L. Tumor Ablation: Common Modalities and General Practices. Tech. Vasc. Interv. Radiol. 16, 192-200 (2013).

2. Perkons, N. R. et al. Electrolytic ablation enables cancer cell targeting through pH modulation. Commun. Biol. 1, 1-10 (2018).

3. Nikfarjam, M., Muralidharan, V. \& Christophi, C. Mechanisms of Focal Heat Destruction of Liver Tumors. J. Surg. Res. 127, 208-223 (2005).

4. Stafford, R. J., Fuentes, D., Elliott, A. A., Weinberg, J. S. \& Ahrar, K. Laser-Induced Thermal Therapy for Tumor Ablation. Crit. Rev. Biomed. Eng. 38, 79-100 (2010).

5. Zhou, Y.-F. High intensity focused ultrasound in clinical tumor ablation. World J. Clin. Oncol. 2, 8 (2011).

6. Martin, R. C. G. Hepatic tumor ablation: cryo versus radiofrequency, which is better? Am. Surg. 72, 391-392 (2006). 
7. Wemyss-Holden, S. A. et al. Electrolytic ablation as an adjunct to liver resection: experimental studies of predictability and safety: Electrolytic ablation as an adjunct to liver resection. Br. J. Surg. 89, 579-585 (2002).

8. Gravante, G. et al. Experimental application of electrolysis in the treatment of liver and pancreatic tumours: Principles, preclinical and clinical observations and future perspectives. Surg. Oncol. 20, 106-120 (2011).

9. Turjanski, P. et al. $\mathrm{pH}$ front tracking in the electrochemical treatment (EChT) of tumors: Experiments and simulations. Electrochimica Acta 54, 6199-6206 (2009).

10. Nordenström, B. Preliminary clinical trials of electrophoretic ionization in the treatment of malignant tumors. IRCS. J. Med. Sci. 6, 537 (1978).

11. Shariat, S. F., Raptidis, G., Masatoschi, M., Bergamaschi, F. \& Slawin, K. M. Pilot study of radiofrequency interstitial tumor ablation (RITA) for the treatment of radio-recurrent prostate cancer. The Prostate 65, 260-267 (2005).

12. Yamakado, K. et al. Radiofrequency Ablation for the Treatment of Unresectable Lung Metastases in Patients with Colorectal Cancer: A Multicenter Study in Japan. J. Vasc. Interv. Radiol. 18, 393-398 (2007).

13. Kim, J. et al. Efficacy and Safety of Radiofrequency Ablation for Treatment of Locally Recurrent Thyroid Cancers Smaller than $2 \mathrm{~cm}$. Radiology 276, 909-918 (2015).

14. Hsiao, C.-W. et al. Photothermal tumor ablation in mice with repeated therapy sessions using NIR-absorbing micellar hydrogels formed in situ. Biomaterials 56, 26-35 (2015).

15. Denisov, A. \& Yeatman, E. Ultrasonic vs. Inductive Power Delivery for Miniature Biomedical Implants. 2010 Int. Conf. Body Sens. Netw. 84-89, https://doi.org/10.1109/BSN.2010.27 (2010).

16. Basaeri, H., Christensen, D. B. \& Roundy, S. A review of acoustic power transfer for bio-medical implants. Smart Mater. Struct. 25, 123001 (2016).

17. Maleki, T. et al. An Ultrasonically-Powered Implantable Micro Oxygen Generator (IMOG). IEEE Trans. Biomed. Eng. https://doi. org/10.1109/TBME.2011.2163634 (2011).

18. Kim, A., Powell, C. R. \& Ziaie, B. An implantable pressure sensing system with electromechanical interrogation scheme. IEEE Trans. Biomed. Eng. 61, 2209-2217 (2014).

19. Zhou, J., Kim, A. \& Ziaie, B. An ultrasonically controlled switching system for power management in implantable devices. Biomed. Microdevices 20, 42 (2018).

20. Kim, A. et al. An Implantable Ultrasonically- Powered Micro-Light-Source ( $\mu$ Light) for Photodynamic Therapy. Nat. Sci. Rep. 9 (2019).

21. Kino, G. S. Acoustic Waves: Devices, Imaging, and Analog Signal Processing. (Prentice Hall, 1987).

22. Ozeri, S. \& Shmilovitz, D. Ultrasonic transcutaneous energy transfer for powering implanted devices. Ultrasonics 50, 556-66 (2010).

23. Song, S. H., Kim, A. \& Ziaie, B. Omnidirectional ultrasonic powering for millimeter-scale implantable devices. IEEE Trans. Biomed. Eng. 62, 2717-2723 (2015).

24. Krimholtz, R., Leedom, D. \& Matthaei, G. Erratum: New equivalent circuits for elementary piezoelectric transducers. Electronics Letters 6, 560 (1970).

25. Morris, P., Hurrell, A., Shaw, A., Zhang, E. \& Beard, P. A Fabry-Pérot fiber-optic ultrasonic hydrophone for the simultaneous measurement of temperature and acoustic pressure. J. Acoust. Soc. Am. 125, 3611-3622 (2009).

26. Maleki, T. et al. An ultrasonically powered implantable Micro-Oxygen generator (IMOG). IEEE Trans. Biomed. Eng. 58, 3104-3111 (2011).

27. Berthois, Y., Katzenellenbogen, J. A. \& Katzenellenbogen, B. S. Phenol red in tissue culture media is a weak estrogen: implications concerning the study of estrogen-responsive cells in culture. Proc. Natl. Acad. Sci. 83, 2496-2500 (1986).

28. Czymek, R. et al. Intrahepatic radiofrequency ablation versus electrochemical treatment in vivo. Surg. Oncol. 21, $79-86$ (2012).

29. Gravante, G. et al. Changes in acid-base balance during electrolytic ablation in an ex vivo perfused liver model. Am. J. Surg. 204, 666-670 (2012).

30. Wang, M. L., Chang, T. C., Charthad, J., Weber, M. J. \& Arbabian, A. The power of sound: miniaturized medical implants with ultrasonic links. In Micro-and Nanotechnology Sensors, Systems, and Applications IX vol. 10194 101940Y (International Society for Optics and Photonics, 2017).

31. Meir, A., Hjouj, M., Rubinsky, L. \& Rubinsky, B. Magnetic Resonance Imaging of Electrolysis. Sci. Rep. 5, 8095 (2015).

32. Holland, R. Resonant Properties of Piezoelectric Ceramic Rectangular Parallelepipeds. Acoust. Soc. Am. J. Of 43, 1-6 (1968).

33. Lamberti, N., Montero Do Espinosa, F. R., Iula, A. \& Carotcnuto, R. Characterization of piezoceramic rectangular parallelepipeds by means of a two-dimensional model. IEEE Trans. Ultrason. Ferroelectr. Freq. Control 48, 113-120 (2001).

34. Ludwig, G. D. The velocity of sound through tissues and the acoustic impedance of tissues. J. Acoust. Soc. Am. 22, 862-866 (1950).

35. Harris, G. \& Services, H. Guidance for Industry and FDA Staff Information for Manufacturers Seeking Marketing Clearance of Diagnostic Ultrasound Systems and Transducers. Food and Drug Administration (2008).

36. Rubinsky, L., Guenther, E., Mikus, P., Stehling, M. \& Rubinsky, B. Electrolytic Effects During Tissue Ablation by Electroporation. Technol. Cancer Res. Treat. 15, NP95-NP103 (2016).

37. Lugnani, F., Macchioro, M. \& Rubinsky, B. Cryoelectrolysis-electrolytic processes in a frozen physiological saline medium. PeerJ 5, e2810 (2017).

38. Sherrit, S., Leary, S. P., Dolgin, B. P. \& Bar-Cohen, Y. Comparison of the Mason and KLM equivalent circuits for piezoelectric resonators in the thickness mode. 1999 IEEE Ultrason. Symp. Proc. Int. Symp. Cat No99CH37027 2, 921-926 (1999).

39. Goss, S. A., Frizzell, L. A. \& Dunn, F. Ultrasonic absorption and attenuation in mammalian tissues. Ultrasound Med. Biol. 5, 181-186 (1979).

\section{Acknowledgements}

We want to acknowledge Dr. Sin Hyung Park for the initial in vitro organoid study. We would also like to thank Mamta Amin and Dr. Mary Barbe in Lewis Katz School of Medicine, Temple University for histology. This work was supported by Temple University start-up funds for Dr. Albert Kim.

\section{Author contributions}

A.K. and B.Z. initiated the project. A.K., S.H.S. and J.Z. designed the device and ultrasonic power system. A.K., S.I. and J.Z. fabricated the device. A.K., S.I., A.K.M., T.P. and R.R. measured the ablation zone estimation. A.K., S.K.L., M.C.P., J.S.P. and J.M.Y. performed and analyzed the in vitro cell experiments. A.K., S.K.L. and M.C.P. performed the ex vivo experiments. A.K. and S.H.S. performed data analysis. A.K., S.H.S. and B.Z. wrote the manuscript.

\section{Competing interests}

The authors declare no competing interests. 


\section{Additional information}

Supplementary information is available for this paper at https://doi.org/10.1038/s41598-020-58090-8.

Correspondence and requests for materials should be addressed to A.K. or B.Z.

Reprints and permissions information is available at www.nature.com/reprints.

Publisher's note Springer Nature remains neutral with regard to jurisdictional claims in published maps and institutional affiliations.

(c) (i) Open Access This article is licensed under a Creative Commons Attribution 4.0 International License, which permits use, sharing, adaptation, distribution and reproduction in any medium or format, as long as you give appropriate credit to the original author(s) and the source, provide a link to the Creative Commons license, and indicate if changes were made. The images or other third party material in this article are included in the article's Creative Commons license, unless indicated otherwise in a credit line to the material. If material is not included in the article's Creative Commons license and your intended use is not permitted by statutory regulation or exceeds the permitted use, you will need to obtain permission directly from the copyright holder. To view a copy of this license, visit http://creativecommons.org/licenses/by/4.0/.

(C) The Author(s) 2020 